\title{
Dielectric Spectroscopy in Ibuprofen-Dioxane Mixture
}

\section{Manoj K. Saini}

School of Physical Sciences, Jawaharlal Nehru University, New Delhi 110067, India

\begin{abstract}
In recent years, the dielectric study of amorphous pharmaceuticals has made a considerable effort towards correlating the molecular mobility with their physical and chemical stability. The molecular mobility of amorphous materials is affected by temperature, additives (such as water) and specific interactions (such as Hydrogen bond). Therefore, to understand the physicochemical instability of amorphous materials, nature of their molecular mobility needs to comprehend.

In this order, the Dielectric and Calorimetric measurements were performed on a mixture of ibuprofen and 1,4-dioxane. The dielectric spectroscopy reveals two relaxation processes, (designated as $\alpha_{\mathrm{D}}$, and $\alpha$ ) in the supercooled region. The spectral shape of $\alpha_{D}$ and $\alpha$ process can be explained satisfactorily throughout the frequency range using Havriliak-Negami $(H N)$ shape function. The $\alpha_{D}$ process is found to be Debye-like (i.e., $\alpha_{H N}=0$ and $\beta_{H N}=$ 1 ) in nature and $\alpha$ process kinetically freezes at $T_{\text {g-onset }}(D S C)$ implies that $\alpha$ - process indeed corresponds to the glass transition event. Both processes are found to be non-Arrhenius in nature. In addition, two secondary relaxation processes (designated as $\beta_{\mathrm{JG}}$ and $\beta$ ) are observed and are comparable with the literature. The activation energy of $\beta$ process indicates that it"s originating from the fluctuations of the side group larger than $-\mathrm{OH}$ group. Also, the calculated fragility index demonstrates that ibuprofen is a fragile glass former.
\end{abstract}

Keywords: Debye relaxation; Dielectric spectroscopy; Ibuprofen; Dioxane

\section{Introduction}

The study of vitrification transition phenomena has always been interested in hydrogen-bonded supercooled liquids composed of small molecules [1-6]. It is known for decades that several monohydroxy alcohols [7-15] and amides [16] hydrogen bonded glass forming systems results in intramolecular and intermolecular hydrogen bonding and behave differently. A study of monohydroxy alcohols reveals that hydrogen-bonded networks aggregate their polar $-\mathrm{OH}$ group in the chain or ring-like structures [9-12]. The aggregation of the chain-like structure shows a Debye relaxation, where the strength of Debye process depends on temperature, pressure, dilution, and confinement volume. On the basis of the position of $-\mathrm{OH}$ group in molecular structure, monohydroxy alcohols can classify in three different groups;

(i) If -OH group is situated at end position (e.g., 2-ethyl-1-hexanol) then observed Debye peak dominates the dielectric loss spectra.

(ii) If -OH group is sterically hindered (e.g., 4-methyl-3-heptanol) then observed Debye peak is weaker. Moreover, if a large cyclic group (e.g., phenyl ring) is located in the molecular structure, it can limit the formation of $\mathrm{H}$-bonding.

If two or more -OH groups are present in the proximity of the same molecule (e.g., glycerol) then Debye peak vanishes [12] or may be hidden under conductivity contribution in the imaginary part of permittivity [13-16]. It is reported that Debye process could not be detected in calorimetric [17], light scattering [18] and nuclear magnetic resonance $[19,20]$. Therefore, dielectric spectroscopy is the most useful technique to study the strength of relaxation processes, which can be discussed in terms of Kirkwood correlation factor [7,21].

Recent $[1,2]$ dielectric studies of supercooled pharmaceuticals are revealing the existence of the Debye-like process in complex molecules, and its presence has been reported in ibuprofen. However, the dielectric strength of the Debye process is found to be very low. Moreover, this Debye-like process is reported to be absent in magnetic resonance or shear mechanical experiments [22].
In this work, the detailed dielectric and calorimetric study of supercooled ibuprofen and its liquid mixtures with 1,4-dioxane at test concentrations is reported. The 1,4-dioxane (also known as p-dioxane) is used as a non-polar [23,24] solute in a solution. The measurements can perform up to $3 \%$ (weight fraction of 1,4-dioxane) because heterogeneous crystallization intervened for higher concentration.

\section{Experimental Section}

\section{Material characterization}

The samples studied are:

(i) Ibuprofen (IBP) $\left(\mathrm{C}_{13} \mathrm{H}_{18} \mathrm{O}_{2}\right)$ (purity $\geq 98 \%$ ) (Molecular weight $(\mathrm{MW})=206.29 \mathrm{~g} / \mathrm{mol})$, also known as $\alpha$-Methyl-4-(isobutyl) phenylacetic acid, ( \pm )-2-(4-Isobutylphenyl) propanoic acid

1,4-dioxane $(\mathrm{DXN})\left(\mathrm{C}_{4} \mathrm{H}_{8} \mathrm{O}_{2}\right)$ (purity $\left.\geq 99.5 \%\right)(\mathrm{MW}=88.11 \mathrm{~g} / \mathrm{mol})$. Short-term inhalation exposure to high levels of 1,4-dioxane may cause nausea, drowsiness, headache, and irritation of the eyes, nose, and throat. The U.S. Environmental Protection Agency (EPA) has classified 1,4-dioxane as likely to be carcinogenic to humans"e by all routes of exposure [25]. The IBP sample is obtained from Sigma-Aldrich co., and DXN is obtained from Fisher Scientific (ExcelaR). Both the samples were dried to remove absorbed water and used without further purification.

\section{Experimental techniques}

Perkin-Elmer sapphire differential scanning calorimeter (DSC) with

*Corresponding author: Manoj K. Saini, School of Physical Sciences, Jawaharlal Nehru University, New Delhi 110067, India, E-mail: manojspsjnu@gmail.com

Received November 22, 2017; Accepted December 22, 2017; Published December 29, 2017

Citation: Saini MK (2017) Dielectric Spectroscopy in Ibuprofen-Dioxane Mixture Pharm Anal Acta 8: 572. doi: 10.4172/2153-2435.1000572

Copyright: (๑) 2017 Saini MK. This is an open-access article distributed under the terms of the Creative Commons Attribution License, which permits unrestricted use, distribution, and reproduction in any medium, provided the original author and source are credited. 
quench-cooling accessory was used for the calorimetric measurements. The DSC cell was calibrated for temperature using indium and mercury as standards. The sample in DSC pans was cooled rapidly at a rate of $10-40 \mathrm{~K} / \mathrm{min}$ to $103 \mathrm{~K}$ in a nitrogen atmosphere, and then DSC scan was made for a heating rate of $10 \mathrm{~K} / \mathrm{min}$.

Frequency domain dielectric measurements were carried out with Agilent E4980A LCR Meter in the frequency range of $20 \mathrm{~Hz}-2 \mathrm{MHz}$. The design of the dielectric cell is similar to the one used before, with the empty cell capacitance $\left(\mathrm{C}_{0}\right)$ of about $15 \mathrm{pF}$. The binary liquids were prepared by mixing well the components using a magnetic stirrer for apparent homogenization. The dielectric cell was then filled with the liquid mixture without entrapping air bubbles, and data was taken during cooling and heating cycles in the argon atmosphere. Before each dielectric scan, the dielectric cell was calibrated with benzene at room temperature. For details of the experimental setup, the reader may consult the earlier publications [4-6].

\section{Data analysis}

To analyzed the complex dielectric function $\varepsilon^{*}(\mathrm{f})=\varepsilon^{\prime}(\mathrm{f})-\varepsilon^{\prime \prime}(\mathrm{f})$, (where $\mathrm{f}$ is frequency, $\varepsilon^{\prime}=$ real, and $\varepsilon^{\prime \prime}=$ imaginary part of the complex permittivity) associated with reorientation of dipoles, imaginary part was fitted to Havriliak-Negami (HN) shape function [26],

$$
\frac{\varepsilon^{*}(f)-\varepsilon_{\infty}}{\varepsilon_{o}-\varepsilon_{\infty}}=\left[1+i\left[\frac{f}{f_{o}}\right]^{1-\alpha_{H N}}\right]^{-\beta_{H N}}
$$

where $f_{0}$ is the average relaxation frequency, $\varepsilon_{0}$ and $\varepsilon_{\infty}$ are the limiting dielectric constant and $\varepsilon_{0}-\varepsilon_{\infty}=\Delta \varepsilon$ is the dielectric strength of the process under consideration, $\alpha_{\mathrm{HN}}$ and $\beta_{\mathrm{HN}}$ are the spectral shape parameters. The parameter $\alpha_{\mathrm{HN}}$ is a measure of the distribution of relaxation times in the sample, and the parameter $\left(1-\beta_{\mathrm{HN}}\right)$ is a measure of cooperation among the molecules. The exact identity of peak loss frequency $\left(f_{m}\right)$ [27] is determined with the help of the estimated values of $\alpha_{\mathrm{HN}}, \beta_{\mathrm{HN}}$ and $\mathrm{f}_{0}$ as,

$$
\begin{aligned}
& f_{m}=f_{o}\left[\frac{k^{\prime}}{\left[\cos \left(\alpha_{H N} \pi / 2\right)-\sin \left(\alpha_{H N} \pi / 2\right) \cdot k^{\prime}\right]}\right]^{1 /\left(1-\alpha_{H N}\right)} \\
& k^{\prime}=\tan \left[\frac{\left(1-\alpha_{H N}\right) \pi}{2\left(1+\beta_{H N}\right)}\right]
\end{aligned}
$$

Where,

The Arrhenius plot of the primary relaxation process has been analysed using the Vogel-Fulcher-Tammann [28] equation given by,

$$
f_{m, \alpha}=f_{o, e} e^{\left[\frac{-B}{T-T_{o}}\right]}
$$

where $\mathrm{T}_{0}$ is the limiting glass transition temperature, $\mathrm{f}_{0^{\prime} \alpha}$ is a constant and $\mathrm{B}=\mathrm{E} / \mathrm{R}$, where $\mathrm{E}$ corresponds to the activation energy. The secondary relaxation process has been analyzed using the Arrhenius equation [29].

$$
f_{m}=f_{o} e^{[-E / R T]}
$$

\section{Results and Discussion}

Prior to the dielectric measurements, DSC measurements were performed on neat IBP and its liquid mixtures with DXN. The pure IBP sample was heated from room temperature to $363.15 \mathrm{~K}$, and an endothermic peak is $\left(\mathrm{T}_{\mathrm{m}}=347.7 \mathrm{~K}\right)$ obtain with an enthalpy of fusion $\left(\Delta \mathrm{H}_{\mathrm{f}}=29 \mathrm{~kJ} / \mathrm{mol}\right)$ (shown in the inset of Figure 1). The molten sample of IBP, is cooled rapidly at a rate of $10-40 \mathrm{~K} / \mathrm{min}$ to $103 \mathrm{~K}$. The vitrified sample is then heated at a rate of $10 \mathrm{~K} / \mathrm{min}$, and the resultant thermal scan (black line) is depicted in Figure 1. The thermal scan shows a glass transition $\left(\mathrm{T}_{\mathrm{g} \text {-onset }}\right.$ ) event at $225.6 \mathrm{~K}$ for IBP. Also, the DSC scan of IBP-DXN ( $\mathrm{x}_{\mathrm{w}}=3 \%$ ) (Where $\mathrm{x}_{\mathrm{w}}$ is the weight fraction of DXN) mixture is shown in Figure 1 and the observed $T_{\text {g-onset }}=215.1$ $\mathrm{K}$ is indicated. Moreover, it has been noticed that neat 1,4-dioxane exhibited a transition (at $\mathrm{T}_{\mathrm{t}}=272 \mathrm{~K}$ ) just a few degrees below its melting temperature (i.e., $\mathrm{T}_{\mathrm{m}}=281.7 \mathrm{~K}$ ), and the observed onset values are in agreement with literature data $[1,7,30]$.

The dielectric measurements were performed in frequency range of $20 \mathrm{~Hz}-2 \mathrm{MHz}$ on IBP and its DXN mixture, and the obtained temperature variation of dielectric permittivity $\left(\varepsilon^{\prime}\right)$ and dielectric loss $(\varepsilon ")$ is shown in Figure 2 (at $\mathrm{f}=1 \mathrm{kHz}$ ), which reveals two (designated as $\alpha_{D}$, and $\alpha$ ) processes in supercooled region. The spectral shape dependence of $\alpha_{D}$, and $\alpha$ relaxation is shown in Figure 3a (at $T=261.2$ $\mathrm{K})$. The dielectric loss spectra of $\alpha_{D}$ and $\alpha$ process can be explained satisfactorily throughout the frequency range using HN Eqn. (1), and the peak loss frequency $\left(f_{m}\right)$ is determined with the help Eqn. (2). In Figure $3 \mathrm{~b}$, the Cole-Cole diagram is shown for the purpose of clarity. Arrhenius plots resolved for both samples are shown in Figure 4. The inverse temperature dependence of peak loss frequency is described well by VFT Eqn. (3) for $\alpha_{D}$, and a process and the details of fit parameters are tabulated in Table 1.

The presence of two relaxation processes in hydrogen-bonded systems in their supercooled regime is not new, similar behavior has been reported for monohydroxy alcohols [7-15] and also, recently observed in neat IBP [1,2]. As discussed above, in such systems, sterically hindered - $\mathrm{OH}$ group can limit the formation of hydrogen bond chain-like structures. Therefore, it's difficult to resolve the Debye process as the structural relaxation dominates. Therefore, in this study a small amount of DXN is added in IBP to disturbed the hydrogen bonds. This results as an enhancement of the dielectric strength of the $\alpha_{D}$ and $\alpha$ relaxation. However, the dielectric strength of $a_{D}$ process is still small in comparison to a process, but it can be resolved more significantly, and this enhancement is strengthening its existence in IBP. The a processes observed are kinetically frozen at $\mathrm{T}_{\text {-onset }}$ (DSC) (Figure 4), indicates that a process indeed corresponds to the glass transition event. Moreover, both processes are found to be non-Arrhenius in nature.

In addition, the dynamic fragility index (m) defined as [5];

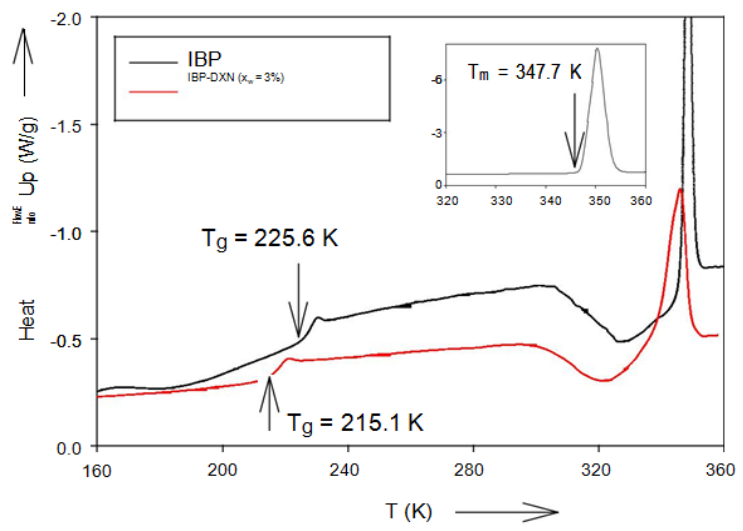

Figure 1: DSC scans taken while heating at a rate of $10 \mathrm{~K} / \mathrm{min}$. for quenched melts of IBP (black line) (sample size $4.3 \mathrm{mg}$ ) and for IBP-DXN $\left(x_{w}=3 \%\right)$ (red line) (sample size $10.5 \mathrm{mg}$ ). Shown in inset is the melting endotherm of neat IBP. 

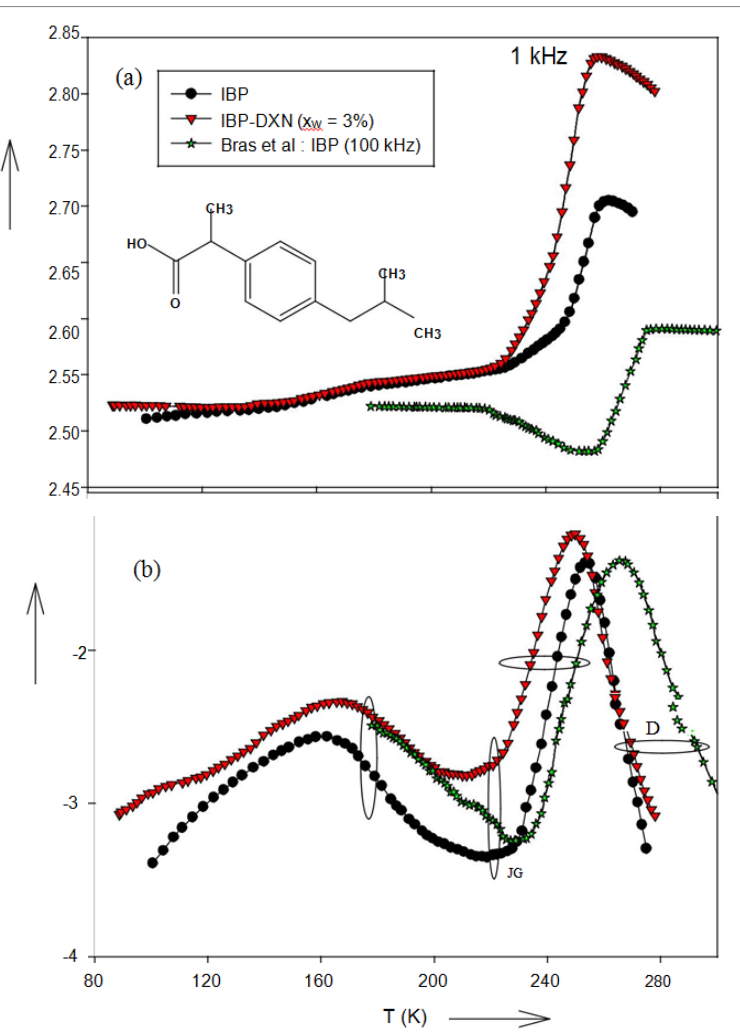

Figure 2: Variation with temperature of (a) the real $\left(\varepsilon^{\prime}\right)$, and (b) the imaginary $\left(\varepsilon^{\prime \prime}\right)$ part of the complex permittivity at a particular frequency $(1 \mathrm{kHz})$ for IBP and IBP-DXN $\left(x_{w}=3 \%\right)$ mixture. Data of pure IBP $(100 \mathrm{kHz})$ are taken from reference [1] for the purpose of comparison. Molecular structure of IBP is shown in the inset.

$$
m=\left.\frac{-d\left(\log f_{m}\right)}{d\left(T_{g-d i e} / T\right)}\right|_{T=T_{g-d i e}}
$$

or

$$
m=\frac{0.4342 B T_{g-d i e}}{\left(T_{g-d i e}-T_{o}\right)^{2}}
$$

has been calculated using VFT-parameters. Fragility measures the rapidity of liquid"s properties, such as viscosity, relaxation time, etc. change as the glassy state is approached. The $m$ value is expected to be smaller for a kinetically strong material than that for a fragile material. The values of $\mathrm{m}$ thus determined for IBP and its mixture are entered in Table 1 . The observed $\mathrm{m}$-index is indicating that IBP is a fragile glass former. Also, the change in fragility values (the mixture is less fragile) is remarkable.

The secondary relaxation processes (designated as $\beta_{\mathrm{IG}}$ and $\beta$ ) observed in dielectric measurement are shown in Figure 4. Albeit, the secondary relaxation process of intermolecular origin (i.e., $\beta_{\mathrm{IG}}$ ), reported in an earlier study [1] is not resolved apparently (due to experimental setup limits and short frequency range). Therefore, the $\mathrm{f}_{\mathrm{m}}$ values corresponding to the $\beta_{\mathrm{IG}}$ process are not stringent and hence, considered with the error bars shown in the Arrhenius diagram is comparable with literature [1]. The $\beta$ process observed is found to be non-Debye in nature, and can be described well by a symmetrical ColeCole function (i.e., Eqn. 1 with $\beta_{\mathrm{HN}}=1$ ) with $\alpha_{\mathrm{HN}} \sim 0.5$ (for IBP), and
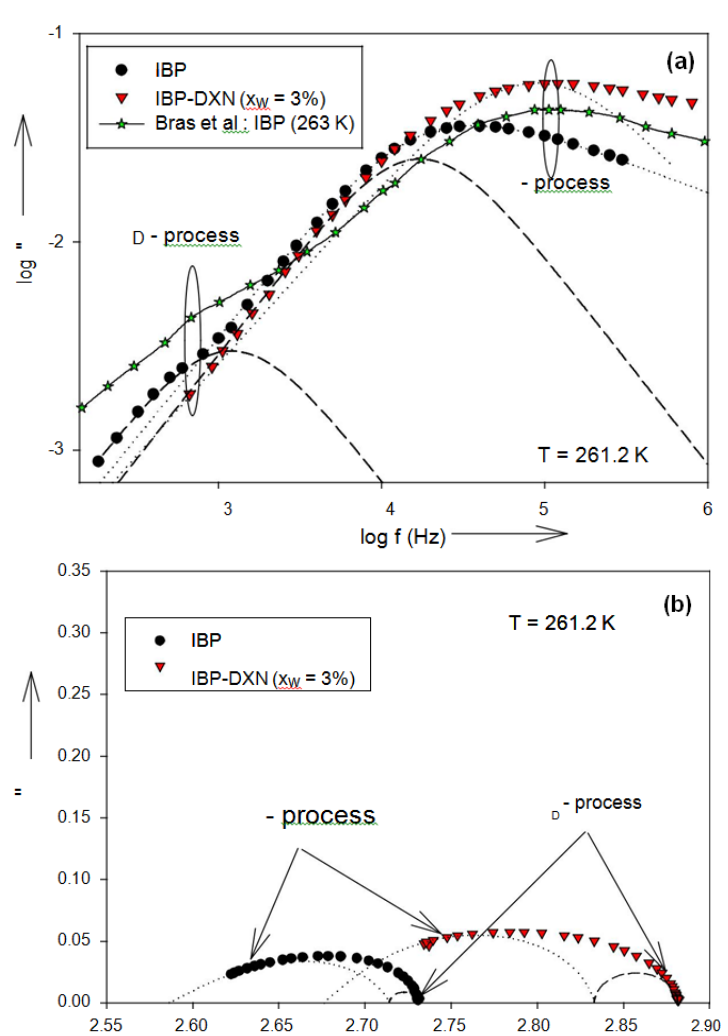

Figure 3: Shown in (a) is the variation of dielectric loss $\left(\varepsilon^{\prime \prime}\right)$ vs. frequency $(f)$ at $\mathrm{T}=261.2 \mathrm{~K}$ in IBP and IBP-DXN $\left(x_{w}=3 \%\right)$. The dashed and dotted lines represent the individual HN Eqn fit corresponds to $\alpha_{\mathrm{D}}$ and $\alpha$ process respectively. Data of pure IBP (at $263 \mathrm{~K}$ ) are taken from reference [1] for the purpose of comparison; and (b) is the Cole-Cole diagram for the purpose of clarity.

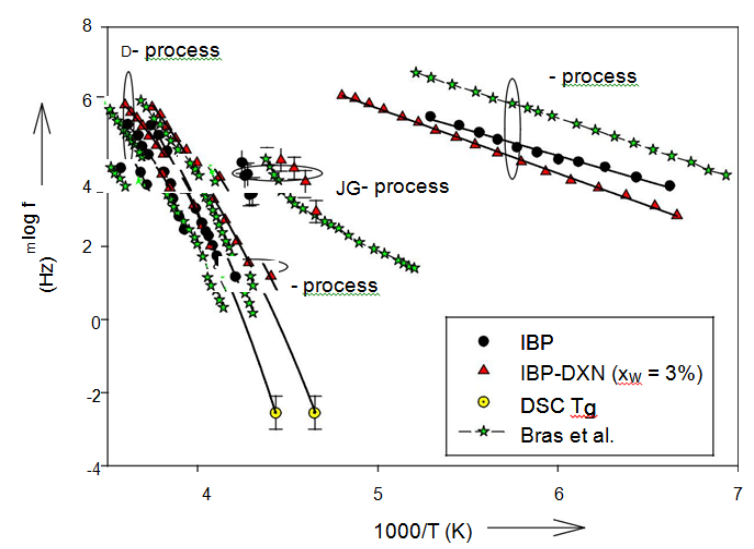

Figure 4: Depicting the Arrhenius plot of IBP and IBP-DXN $\left(x_{w}=3 \%\right)$. The thick line along the $\alpha_{\mathrm{D}}$ and $\alpha$ process is corresponds to Eqn. (3), and that along the $\beta$ process is corresponds to Eqn. (4). The $f$ values corresponding to $\beta$ process are not stringent and hence, considered with the error bars shown in the Arrhenius diagram. Data of pure IBP are taken from ref [1] are also shown for the purpose of comparison. Additionally, shown in the diagram is the enthalpy relaxation frequency at $T$, determined by the DSC which corresponds to an approximate $\log f_{m}(\mathrm{~Hz})=-2.55 \pm 0.45$.

$a_{\mathrm{HN}} \sim 0.6$ (for IBP-DXN ( $\left.\mathrm{x}_{\mathrm{w}}=3 \%\right)$ mixture). The inverse temperature dependence of peak loss frequency is described well by Arrhenius Eqn. (4), and the corresponding details are tabulated in Table 1. The 


\begin{tabular}{|c|c|c|c|c|c|c|c|c|}
\hline \multirow[b]{2}{*}{ Sample } & \multirow[b]{2}{*}{ Process } & \multicolumn{3}{|c|}{ VFT Eqn. (3) } & \multicolumn{2}{|c|}{$T_{g-d i e^{*}}$} & \multicolumn{2}{|c|}{ Arrhenius Eqn. (4) } \\
\hline & & $\log f_{o}(\mathrm{~Hz})$ & B (K) & $T_{0}(\mathrm{~K})$ & (K) & m & $\underset{(\mathrm{Hz})}{\log \boldsymbol{f}_{o}}$ & $\begin{array}{c}E_{\beta}(\mathbf{k J} / \\
\mathrm{mol})\end{array}$ \\
\hline \multirow{3}{*}{ IBP } & $\alpha D$ & 9.1 & 439.8 & 225.8 & - & - & - & - \\
\hline & $\alpha-$ & 19.1 & 3581.9 & 153.7 & 224.1 & 70 & - & - \\
\hline & $\beta-$ & - & - & - & - & - & 12.9 & 27 \\
\hline IBP-DXN & $\alpha D-$ & 10.7 & 824 & 204.3 & - & - & - & - \\
\hline \multirow{2}{*}{$\left(x_{w}=3 \%\right)$} & $\alpha-$ & 17.2 & 3173.5 & 145.3 & 213.5 & 63 & - & - \\
\hline & $\beta-$ & - & - & - & - & - & 14.6 & 30.9 \\
\hline
\end{tabular}

$T_{g-\text {-die }}$ is the temperature at which the $f_{m}$ value is $\sim 10^{-3} \mathrm{~Hz}$

Table 1: Details of the various fit parameters are tabulated.

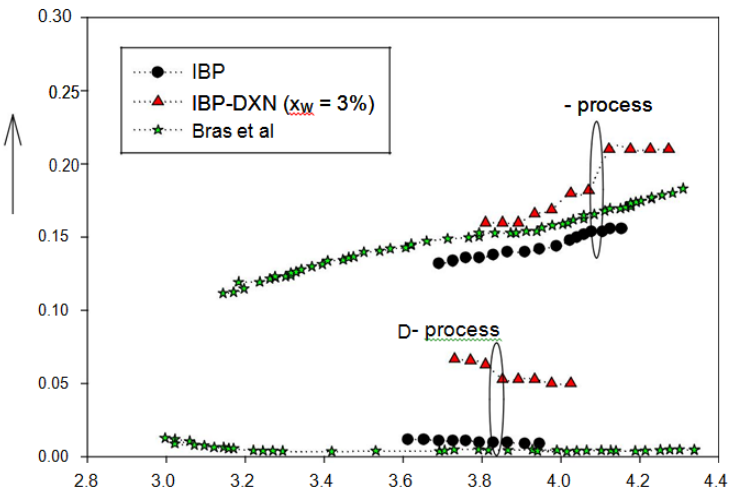

Figure 5: Depicting the variation of dielectric strength $(\Delta \varepsilon)$ with inverse of temperature for $\alpha_{\mathrm{D}}$ and $\alpha$ processes in IBP and IBP-DXN $\left(x_{w}=3 \%\right)$. Data of pure IBP are taken from ref [1] for the purpose of comparison. The dotted lines are used to guide eyes.

estimated activation energies $\left(\mathrm{E}_{\beta}\right)$ are comparable with literature data [1]. This agreement of activation energies with literature is indicating that the observed $\beta$ process is intramolecular in nature and originates from the fluctuations of the side group larger than - $\mathrm{OH}$ group.

From Figure 5, it is evident that the dielectric strength (i.e., $\Delta \varepsilon$ ) of $\alpha_{D}$ and $\alpha$ process is enhanced after adding a small amount of DXN. The dielectric strength is found to follow a linear correlation with temperature for $\alpha_{D}$ process. In contrast, an inverse correlation is observed for a process, which is well known for conventional glass formers. Such behaviour of $\Delta \varepsilon$ with temperature can be understood in terms Fuoss-Kirkwood correlation factor $\left(\mathrm{g}_{\mathrm{k}}\right)$, which can be estimated by Onsager- Kirkwood-Frohlich equation [29-31].

$$
g_{k}=\left(\varepsilon_{o}-\varepsilon_{\infty}\right) \frac{9 M k_{B} T\left(2 \varepsilon_{o}+\varepsilon_{\infty}\right)}{4 \pi N_{A} \rho \mu_{o}^{2} \varepsilon_{o}\left(\varepsilon_{\infty}+2\right)^{2}}
$$

In this formula $\varepsilon_{0}$ is the equilibrium dielectric constant, $\varepsilon_{\infty}$ is limiting high frequency permittivity due to induced polarization, $\mathrm{N}_{\mathrm{A}}$ is the Avogadro"s number, $\mathrm{M}$ is the molecular weight, $\rho$ is the density, $\mu_{0}$ is the dipole moment of the isolated molecule, $g_{k}$ is FuossKirkwood correlation factor, and $\mathrm{k}_{\mathrm{B}}$ is the Boltzmann constant. FuossKirkwood correlation factor $g_{k}$ is a measure of the correlation of the neighboring dipole vectors. When $\mathrm{g}_{\mathrm{k}}>1$, indicates the tendency of parallel alignment of neighboring dipoles and $g_{k}<1$, indicates the tendency of anti-parallel alignment of neighboring dipoles. Here, the small values of $\Delta \varepsilon$ are in agreement that hydrogen bonding in these samples favors an anti-parallel correlation of the neighboring dipoles $[1,7,32]$. Some information of the dipole moment $\left(\mu_{0}\right)$ is necessary to understand the dielectric strength variation. Unfortunately, the experimental value of $\mu_{0}$ is not available for neat IBP. Although, it is reported in the literature [33] that the dipole moment of IBP is mostly originated from $\mathrm{O}=\mathrm{C}-\mathrm{O}-\mathrm{H}$ group. According to Bras et al. [1] the Molecular Dynamics (MD) simulation reveals a strong tendency of IBP to form noncovalent molecular aggregates such as dimer $\left(\mu_{0}=1.29 \mathrm{D}\right.$ to $2.15 \mathrm{D})$ and trimer $\left(\mu_{0}=1.68 \mathrm{D}\right.$ to $\left.2.50 \mathrm{D}\right)$ either cyclic or linear, where cyclic structures were found to have a smaller dipole moment and less $\mathrm{g}_{\mathrm{k}}$ "value ( $\sim 0.3$ (for cyclic) and $\sim 0.8$ (for linear)) at $\mathrm{T}=360 \mathrm{~K}$. Also, it is suggested that hydrogen bonded cyclic structures might be associated with Debye process in IBP. Later, Affouard et al. [2] attempts to reveal the origin of the Debye type process in IBP and performed the MD simulation on racemic IBP. This simulation suggests that the Debye type process is dominated by the internal „cis $\left(\mu_{0}=1.6 \mathrm{D}\right)$ " to "trans $\left(\mu_{0}=4.6 \mathrm{D}\right)$ " conversion of the $\mathrm{O}=\mathrm{C}-\mathrm{O}-\mathrm{H}$ group coupled to the change of the intermolecular linear/cyclic hydrogen-bonded structures. Also, it is found that the linear structures (dimers/trimers) are favoring the conversion and stabilize to the trans isomer. However, for internal dipolar reorientations (i.e. cis-trans conversion in the effective potential of neighboring dipoles), the breaking and reforming of intermolecular hydrogen bonds is necessary.

\section{Conclusions}

The dielectric measurements of pure ibuprofen and its mixture with 1,4-dioxane exhibit two relaxations, designated as $\alpha_{D}$, and $a$ processes. The $\alpha_{D}$ process is found to be Debye type in nature, and $\alpha$ process kinetically freezes at $T_{g-o n s e t}$ (DSC) implies that a process indeed corresponds to the glass transition event. The spectral shape of $\alpha_{D}$, and $\alpha$ processes can be explained satisfactorily throughout the frequency range using $\mathrm{HN}$ shape function, and are found to be nonArrhenius in nature. The enhancement of dielectric strength of both processes is remarkable. Although, the dielectric strength of $\alpha_{D}$ process is still small in comparison to a process, but it can be resolved more significantly, and this enhancement is strengthening its existence in IBP. The dielectric strength is found to follow a linear correlation with temperature for $\alpha_{D}$ process. In contrast, an inverse correlation is observed for process, which is well known for conventional glass formers. The secondary relaxation processes are comparable with the literature [1], and the activation energy of $\beta$ process indicates that its originating from the fluctuations of the side group larger than - $\mathrm{OH}$ group. Also, the calculated fragility index demonstrates that ibuprofen is a fragile glass former. Moreover, the experimental evidence of the origin of $\alpha_{D}$ process in IBP, needs to be probe further.

\section{Acknowledgment}

The author wishes thank to Prof. S.S.N. Murthy for his valuable guidance as well his kind encouragement, and thanks to Department of Science and Technology for the financial support.

\section{References}

1. Bras AR, Noronha JP, Antunes AMM, Cardoso MM, Schonhals A, et al. (2008) Molecular Motions in Amorphous Ibuprofen As Studied by Broadband Dielectric Spectroscopy. J Phys Chem B 112: 11087-11099.

2. Affouard F, Correia NT (2010) Debye Process in Ibuprofen Glass- Forming Liquid: Insights from Molecular Dynamics Simulation. J Phys Chem B 114 11397-11402.

3. Bhattacharya S, Suryanarayanan R (2009) Local mobility in amorphous pharmaceuticas characterization and implications on stability. J Pharma Sci 98: 2935-2953.

4. Saini MK, Murthy SSN (2014) Study of glass transition phenomena in the supercooled liquid phase of methocarbamol, acetaminophen and mephenesin. Thermochemica Acta 575: 195-205.

5. Saini MK, Murthy SSN (2015) Glass Formation in Binary Solutions of Acetaminophen with Guaifenesin and Mephenesin. J Solution Chem 44: 17231748. 
6. Saini MK, Murthy SSN (2016) Acetaminophen, Methocarbamol, Guaifenesin and Mephenesin in Propylene Glycol: Solubility and Phase Behaviour for Use in Medical Industry. Pharm Anal Acta 7: 1000493.

7. Murthy SSN (1996) Dielectric relaxation in monohydroxy alcohols and its connection to the glass transition process. J Phys Chem 100: 8508- 8517.

8. Murthy SSN, Tyagi M (2002) Experimental study of the high frequency relaxation process in monohydroxy alcohols. J Chem Phys 117: 3837-3847.

9. Gainaru C, Kastner S, Mayr F, Lunkenheimer P, Schildmann S, et al. (2011) Hydrogen-bond equilibria and lifetimes in a monohydroxy alcohol. Phys Rev Let 107: 118304.

10. Singh LP, Richert R (2012) Watching hydrogen-bonded structures in an alcohol convert from rings to chains. Phys Rev Let 109: 167802.

11. Pawlus S, Wikarek M, Gainaru C, Poluch M, Bohmer R (2013) How do high pressures change the Debye process of 4-methyl-3-heptanol? J Chem Phys 139: 064501

12. Gainaru C, Meier R, Schildmann S, Lederle C, Hiller W, et al. (2010) NuclearMagnetic-Resonance measurements reveal the origin of the Debye process in monohydroxy alcohols. Phys Rev Let 105: 258303.

13. Bergman R, Jansson H, Swenson J (2010) Slow Debye-type peak observed in the dielectric response of polyalcohols. J Chem Phys 132: 044504.

14. Richert R (2010) Dynamics of glass-forming liquids. XIV. A search for ultraslow dielectric relaxation in glycerol. J Chem Phys 133: 074502.

15. Bergman R, Jansson H, Swenson J (2011) Response to Comment on Slow Debye-type peak observed in the dielectric response of polyalcohols. J Chem Phys 134: 037102

16. Wang LM, Richert $R$ (2005) Identification of dielectric and structural relaxations in glass-forming secondary amides. J Chem Phys 123: 054516.

17. Huth H, Wang LM, Schick C, Richert R (2007) Comparing calorimetric and dielectric polarization modes in viscous 2-ethyl-1-hexanol. J Chem Phys 126: 104503.

18. Hansen C, Stickel F, Berger T, Richert R, Fischer EW (1997) Dynamics of glass-forming liquids. III. Comparing the dielectric $\alpha$ and $\beta$ relaxation of 1-propanol and o-terphenyl. J Chem Phys 107: 1086-1093.

19. Lederle C, Hiller W, Gainaru C, Bohmer R (2011) Diluting the hydrogen bonds in viscous solutions of $n$-butanol with $n$-bromobutane: II. A comparison of rotational and translational motions. J Chem Phys 134: 064512.
20. Schildmann S, Reiser A, Gainaru R, Gainaru C, Bohmer R (2011) Nuclear magnetic resonance and dielectric noise study of spectral densities and correlation functions in the glass forming monoalcohol 2-ethyl- 1-hexanol. J Chem Phys 135: 174511

21. Kirkwood JG (1939) The Dielectric Polarization of Polar Liquids. J Chem Phys 7: $911-919$

22. Bauer S, Storek M, Gainaru C, Zimmermann H, Bohmer R (2015) Molecular Motions in Supercooled and Glassy lbuprofen: Deuteron Magnetic Resonance and High-Resolution Rheology Study. J Phys Chem B 119: 5087-5095.

23. Kumar S, Periyasamy $P$, Jeevanandham $P$ (2011) Dielectric relaxation studies of binary liquid mixtures of a few glycols with 1,4-Dioxane. Int $\mathrm{J}$ Chem Tech Res 3: 369-375.

24. Schwingel $\mathrm{CH}$, Williams EW (1934) The Electric Moment of Dioxane. Lab Phys Chem, University of Wisconsin Madison, Wisconsin 56: 653-654.

25. United State Environmental Protection Agency (2014) Technical Fact Sheet1,4-Dioxane. EPA 505-F-14-011.

26. Havriliak S, Negami S (1966) A Complex Plane Analysis of $\alpha$ - Dispersions in Some Polymer Systems. J Pol Sci Part C 117: 99-117.

27. Murthy SSN (1997) Phase Behavior of the Supercooled Aqueous Solutions of Dimethyl Sulfoxide, Ethylene Glycol, and Methanol As Seen by Dielectric Spectroscopy. J Phys Chem 101: 6043-6049.

28. Murthy SSN (1992) Molecular Dynamics in Supercooled Liquids: A study of the relaxation in binary solutions. J Molecular Liquids 51: 197-217.

29. Hill NE, Vaughan WE, Price AH, Davies M (1969) Dielectric Properties and Molecular Behaviour. London: Van Nostrand Reinhold.

30. Jacobs CJ, Parks G (1934) Thermal Data on Organic Compounds. XIV. Some Heat Capacity, Entropy and Free Energy Data for Cyclic Substances. Stanford University, California 56: 1513-1517.

31. Dannhauser W (1968) Dielectric Study of Intermolecular Association in Isomeric Octyl Alcohols. J Chem Phys 48: 1911-1917.

32. Johari GP, Kim S, Shanker RM (2007) Dielectric Relaxation and Crystallization of Ultraviscous Melt and Glassy States of Aspirin, Ibuprofen, Progesterone, and Quinidine. J Pharm Sci 96: 1159-1175.

33. Vueba ML, Pina ME, Veiga F, Sousa JJ, Batista De Carvalho LAE (2008) Conformational Stability of Ibuprofen: Assessed by DFT Calculations and Optical Vibrational Spectroscopy. J Pharm Sci 97: 845-859. 